Article

\title{
A Comparison of Two Single-Stranded DNA Binding Models by Mutational Analysis of APOBEC3G
}

\author{
Keisuke Shindo ${ }^{\dagger}$, Ming Li, Phillip J. Gross, William L. Brown, Elena Harjes, Yongjian Lu, \\ Hiroshi Matsuo * and Reuben S. Harris *
}

Department of Biochemistry, Molecular Biology and Biophysics, Institute for Molecular Virology, University of Minnesota, Minneapolis, MN 55455, USA;

E-Mails: shind009@kuhp.kyoto-u.ac.jp (K.S.); mingli@umn.edu (M.L.); gros0287@umn.edu (P.J.G.); brown344@umn.edu (W.L.B.); harje002@umn.edu (E.H.); luxxx079@umn.edu (Y.L.)

$\dagger$ Present address: Department of Hematology and Oncology, Graduate School of Medicine, Kyoto University, Kyoto, Kyoto 606-8507, Japan.

* Authors to whom correspondence should be addressed; E-Mails: matsu029@umn.edu (H.M.); rsh@umn.edu (R.S.H.); Tel.: +1-612-625-6100.

Received: 23 May 2012; in revised form: 1 July 2012 / Accepted: 14 July 2012 /

Published: 2 August 2012

\begin{abstract}
APOBEC3G is the best known of several DNA cytosine deaminases that function to inhibit the replication of parasitic genetic elements including the lentivirus HIV. Several high-resolution structures of the APOBEC3G catalytic domain have been generated, but none reveal how this enzyme binds to substrate single-stranded DNA. Here, we constructed a panel of APOBEC $3 \mathrm{G}$ amino acid substitution mutants and performed a series of biochemical, genetic, and structural assays to distinguish between "Brim" and "Kink" models for single-strand DNA binding. Each model predicts distinct sets of interactions between surface arginines and negatively charged phosphates in the DNA backbone. Concordant with both models, changing the conserved arginine at position 313 to glutamate abolished both catalytic and restriction activities. In support of the Brim model, arginine to glutamate substitutions at positions 213, 215, and 320 also compromised these APOBEC3G activities. Arginine to glutamate substitutions at Kink model residues 374 and 376 had smaller effects. These observations were supported by A3G catalytic domain-ssDNA chemical shift perturbation experiments. The overall data set is most consistent with the Brim model for single-stranded DNA binding by APOBEC3G.
\end{abstract}


Keywords: APOBEC3G; DNA cytosine deamination; HIV restriction; single-stranded DNA; structure-guided mutagenesis

\section{Introduction}

APOBEC3G (A3G) is one of seven human APOBEC3 subfamily members, which all belong to a larger family of polynucleotide cytosine deaminases that includes the antibody gene diversification enzyme AID and the $A P O B$ mRNA editing enzyme APOBEC1 (reviewed by [1,2]). A3G is one of the most intensively studied family members due to its potent HIV-1 (hereafter HIV) restriction activity (reviewed by [3-5]). A3G restricts HIV replication by packaging into assembling viral particles, travelling with virions until a new target cell is breached, and then deaminating cytosines to uracils in nascent viral cDNA during retrovirus reverse transcription. Uracils bind to adenines during plus strand synthesis which results in hallmark G-to-A hypermutations in the viral genome. Although three other subfamily members also contribute to HIV restriction and hypermutation [6,7], A3G was among the first to be discovered [8-10] and the first to yield to biochemical and structural studies [11-17]. A3G has therefore become the prototype for understanding the molecular mechanisms of substrate recognition and catalysis by the broader family of DNA deaminases.

A3G consists of two phylogenetically distinct zinc-coordinating domains, an amino(N)-terminal Z2-type domain (residues 1-196) and a carboxy(C)-terminal Z1-type domain (residue 197-384) [2]. Each domain has a characteristic $\mathrm{H}-\mathrm{x}_{1}-\mathrm{E}-\mathrm{x}_{23-28}-\mathrm{C}-\mathrm{x}_{2-4}-\mathrm{C}$ zinc-coordinating motif that can be distinguished by a number of amino acid and activity differences. The amino-terminal Z2 domain is incapable of catalysis, but it is largely responsible for cytoplasmic localization, interacting with HIV Vif (the virus' natural counterdefense), and binding RNA and single-stranded (ss)DNA (e.g., [11,14,18-21] and this study). The C-terminal half of the protein provides substrate lysines for Vif-dependent poly-ubiquitination, dictates the local dinucleotide deamination preference, and catalyzes ssDNA

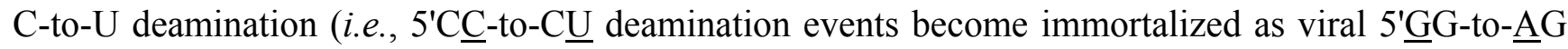
hypermutations) [20,22-26]. As such, the $\mathrm{C}$-terminal domain is critical for recognizing specific regions of ssDNA, catalyzing C-to-U deamination, and blocking HIV replication. Neither domain alone is sufficient for HIV restriction.

Several structures of the A3G catalytic domain have been obtained using NMR [13,16,27] and X-ray crystallography $[12,17,28]$. These studies have led to two distinct models of how ssDNA molecules may position during C-to-U deamination [12,16,27] (Figure 1). The "Brim" model hypothesizes that the positive charges of four arginines R213, R215, R313 and R320 serve to bind the negatively charged phosphodiester backbone such that the target cytosine positions appropriately in the catalytic pocket [16,27]. The "Kink" model proposes that ssDNA bends at the active site and interacts with a partly overlapping set of surface residues including arginines R313, R320, R374, and R376 and asparagine N244 [12]. Key differences include Brim model residues R213 and R215 and Kink model residues $\mathrm{R} 374$ and $\mathrm{R} 376$. 
Figure 1. APOBEC3G catalytic domain DNA binding models. (a) A ribbon schematic of the A3G191-384-2K3A crystal structure (3IR2) depicting amino acid side chains relevant to the present studies. (b) The predicted positioning of ssDNA (dashed line) according to the Brim model. (c) The predicted positioning of ssDNA (dashed line) according to the Kink model.

(a)

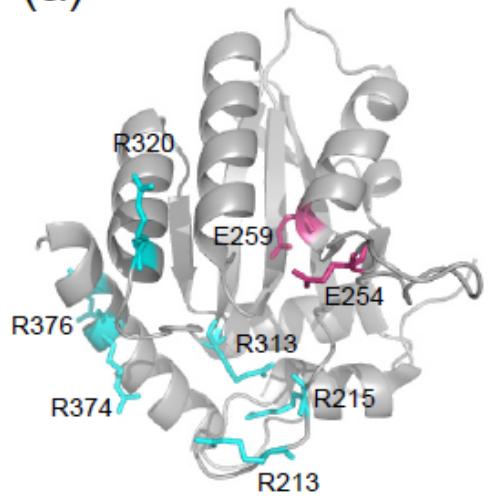

(b)

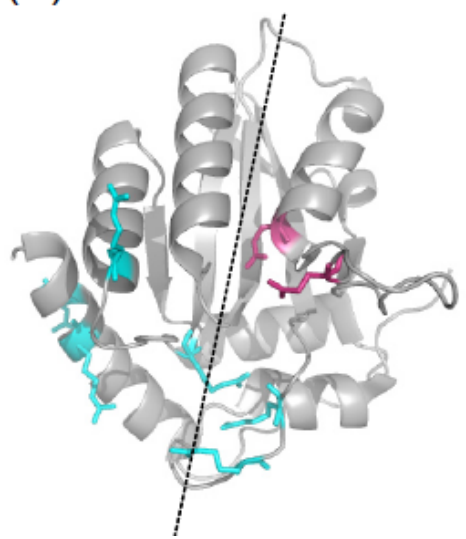

(c)

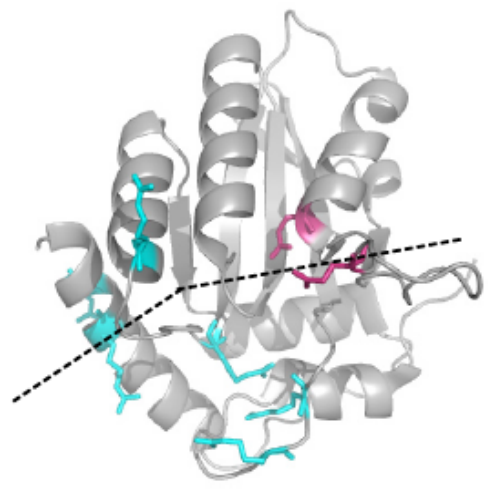

To distinguish between these two DNA binding models, we constructed a series of A3G mutants with charge altering amino acid substitutions at each of these positions. All mutants were tested in parallel in ssDNA C-to-U deamination assays, electrophoretic mobility shift assays (EMSAs), and HIV restriction experiments. Residues R215 and R313 proved essential for catalysis and HIV restriction. R213, R320, and R374 had intermediate phenotypes, and R376 was dispensable. These observations were supported by NMR spectroscopy chemical shift perturbation experiments with isotope-labeled A3G catalytic domain and varying concentrations of unlabeled ssDNA. We conclude that the Brim model provides a more accurate representation of ssDNA binding by the $\mathrm{A} 3 \mathrm{G}$ catalytic domain.

\section{Results and Discussion}

\subsection{DNA Deaminase Activity Experiments}

Wildtype and mutant A3G proteins were expressed in HEK293T cells with a C-terminal multipurpose tag consisting of a Strep epitope, a Tobacco Etch Virus (TEV) protease cleavage site, and two copies of the Ig-binding domain of Protein A (A3G-STP). IgG sepharose was used for affinity purification, and TEV protease was used to release A3G-S from the solid support into the supernatant. The yield and purity of each protein was comparable as determined by SDS-PAGE fractionation and silver staining (Figure 2a). Subtle migration differences were attributable to introduced charge changes.

Next, we examined activity of the purified proteins using a fluorescence-based DNA C-to-U deaminase assay (Figure 2b). We used a 69 nucleotide ssDNA covalently linked to Alexa488 at the 5'-end. Deamination of the A3G-preferred 5'-CCC motif to 5'-CCU (or 5'-CUC or 5'-CUU) creates a substrate for uracil DNA glycosylase (UDG), which in turn creates an abasic site susceptible to hydrolytic cleavage. Thus, because UDG and $\mathrm{NaOH}$ are present in excess, the visual appearance of 32 (primary target) and 31 (secondary target) nucleotide fragments provides a quantitative measure of deaminase activity. 
Figure 2. Deamination assays in vitro and in bacteria. (a) Silver-stained gel of affinity purified $\mathrm{A} 3 \mathrm{G}$ and derivatives. Bovine serum albumin (BSA) was used for quantification. (b) Gel image of ssDNA deamination substrate and products produced by incubation with the indicated A3G proteins in vitro. (c) Gel image of ssDNA deamination substrate and products produced by incubation with varying amounts of the indicated $A 3 \mathrm{G}$ proteins in vitro. $(\mathbf{d}) \mathrm{Rif}^{\mathrm{R}}$ mutation data for the indicated $\mathrm{A} 3 \mathrm{G}$ proteins expressed in E. coli. Each $\mathrm{X}$ corresponds to data from one independent overnight culture, median mutation frequencies are shown, and $\mathrm{A} 3 \mathrm{G}$ protein levels are shown in the immunoblot below.

(a)

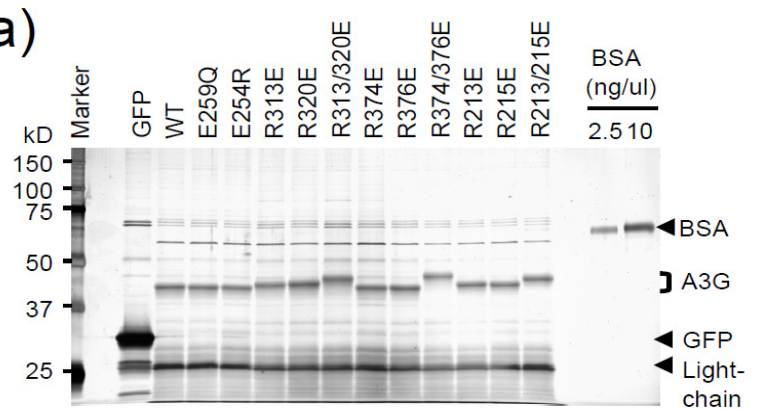

(b)

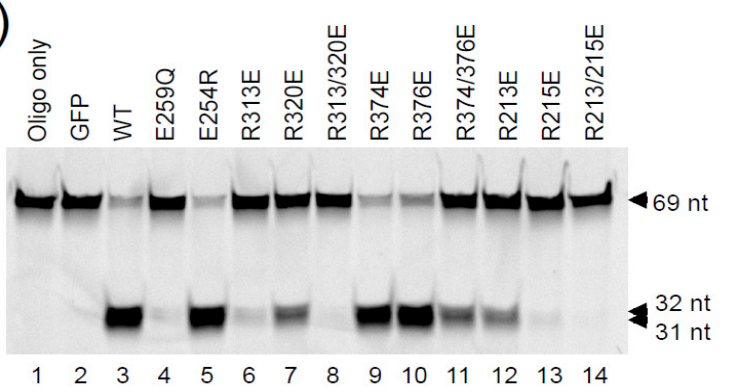

(c)

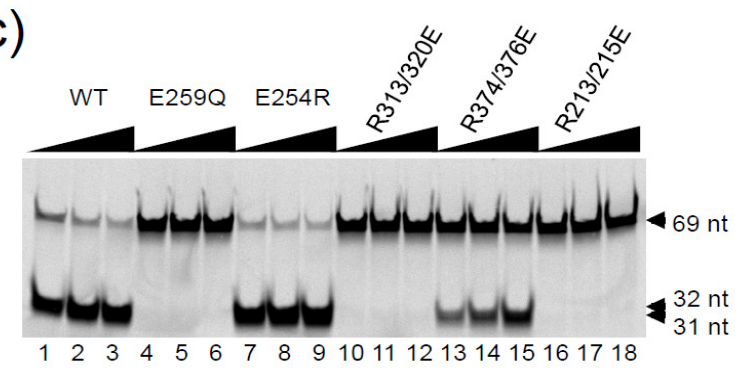

(d)

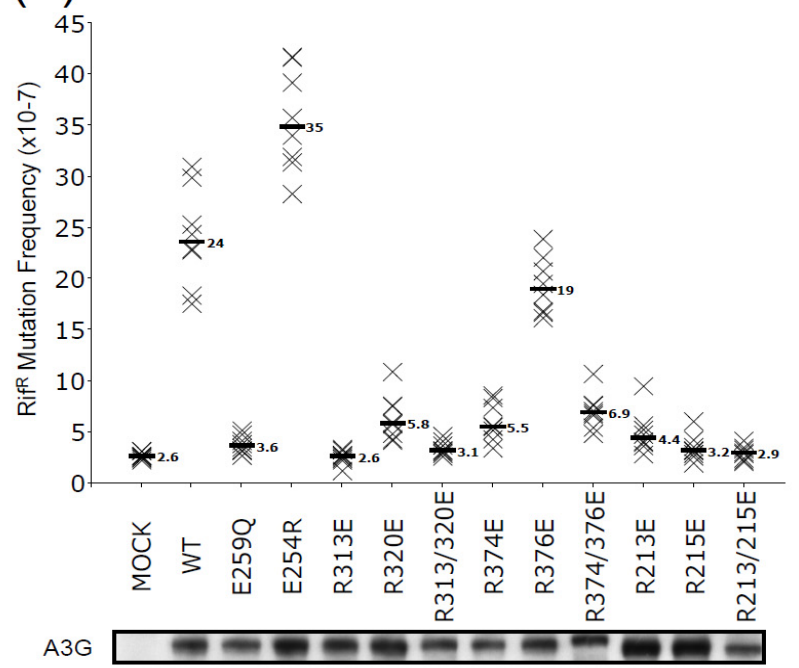


Wildtype A3G and the E254R derivative showed strong activity with almost all of the 69 nucleotide substrate converted to shorter reaction products (Figure 2b, lanes 3 and 5). GFP or A3G-E259Q, a well-characterized catalytic mutant [26,29,30], had no activity indicating that other nucleic acid processing activities were not co-purifying (Figure 2b, lanes 2 and 4). Single amino acid substitution mutants, R313E or R215E, or double mutants with these substitutions lost almost all activity (Figure 2b, lanes 6, 8, 13 and 14). The faint deamination products apparent in these lanes may be due to a low rate of activity. In contrast, R374E and R376E showed activity similar to wildtype A3G (Figure 2b, lanes 3, 9 and 10), and the R374E/R376E double mutant had impaired but still obvious deaminase activity (Figure 2b, lane 11). Similar results were obtained in a titration experiment, with arginine pairs 313/320 and 213/215 proving more important than 374/376 (Figure 2c).

Another sensitive readout for intrinsic A3G DNA C-to-U deaminase activity is the E. coli rifampicin-resistance $\left(\operatorname{Rif}^{\mathrm{R}}\right)$ mutation assay [8]. To corroborate the biochemical data described above, all A3G constructs were cloned as untagged cDNAs into the bacterial vector pTrc99A and transformed into UDG-deficient $E$. coli. The resulting colonies were outgrown into saturated overnight cultures and subjected to selection on plates containing rifampicin ( $n=8$ independent cultures per condition). As reported originally [8], wildtype A3G caused a 10-fold increase in median Rif $^{\mathrm{R}}$ mutation frequency in comparison to E. coli expressing an empty vector control or the E259Q catalytic mutant (Figure 2d). The E254R mutant showed slightly higher activity, which may reflect slightly higher expression levels. As above, any single or double mutant with R313E or R215E was completely inactive. In contrast, R376E had near wildtype activity and R374E or the R374E/R376E double mutant had activity 2-fold above background. Taken together, these data demonstrate that R313 and R215 are essential for catalytic activity, R320, R213, and R374 are influential, and R376 is dispensable.

\subsection{HIV Restriction Experiments}

A3G is a potent inhibitor of Vif-deficient HIV replication, and the majority of its restriction capacity is attributable to deaminase activity [26,31-35]. We therefore asked whether the biochemical and genetic deaminase activity phenotypes of the aforementioned mutants correlate with HIV restriction activity. Vif-deficient HIV-GFP was produced in the presence of each A3G construct by transient transfection of HEK293T cells. The resulting cell-free supernatants each contained equivalent levels of virus as judged by anti-p24 (CA) immunoblots (Figure 3a). Infectivity was quantified by incubating equivalent volumes of each supernatant with target cells and monitoring infectivity $44 \mathrm{~h}$ later by flow cytometry for GFP signal. As expected, wildtype A3G and E254R both strongly restricted virus infectivity, whereas the E259Q catalytic mutant was impaired (Figure 3a; note that low ratios of $\mathrm{A} 3 \mathrm{G}$ to proviral plasmid were used in each transfection to try to minimize deaminase-independent effects [29,35-37]). A3G-dependent restriction was also seriously compromised by R313E or R215E, which were modestly exacerbated by adjacent R320E or R213E substitutions, respectively. In contrast, Vif-deficient HIV was still restricted fully by R376E, strongly by R374E, and intermediately by the combination of these two substitutions. Similar observations were made in dose response experiments, with the R313E/R320E and R213E/R215E restriction phenotypes being indistinguishable from the $\mathrm{E} 259 \mathrm{Q}$ catalytic mutant (with some restriction due to deaminase-independent over-expression [29,35-37]) and R374E/R376E causing intermediate levels of 
restriction (Figure 3b). All mutants packaged similarly and localized indistinguishable from wildtype A3G to the cytoplasmic region of transfected cells (Figure 3a,b, and fluorescent microscopy data not shown). The HIV restriction phenotypes presented here correlate directly with the deaminase activity data presented above.

Figure 3. HIV restriction by $\mathrm{APOBEC} 3 \mathrm{G}$ and mutant derivatives. (a) A histogram reporting the infectivity of Vif-deficient HIV-1 produced in the presence of a GFP control expression vector (Mock), wildtype A3G-GFP (WT), or the indicated A3G mutants (1 part A3G plasmid or vector control to 30 parts proviral DNA cocktail; see Methods for details). Representative immunoblots are shown below for cell lysates and viral particles. A3G-GFP, Tubulin and anti-p24 (CA) were detected using anti-GFP, anti-tubulin and anti-p24 antibodies, respectively. (b) Dose response infectivity data analogous to panel 'a', excepted A3G-GFP or the indicated mutant expression construct was titrated into each transfection at the indicated $\mathrm{A} 3 \mathrm{G}$ :proviral plasmid cocktail ratio.

(a)

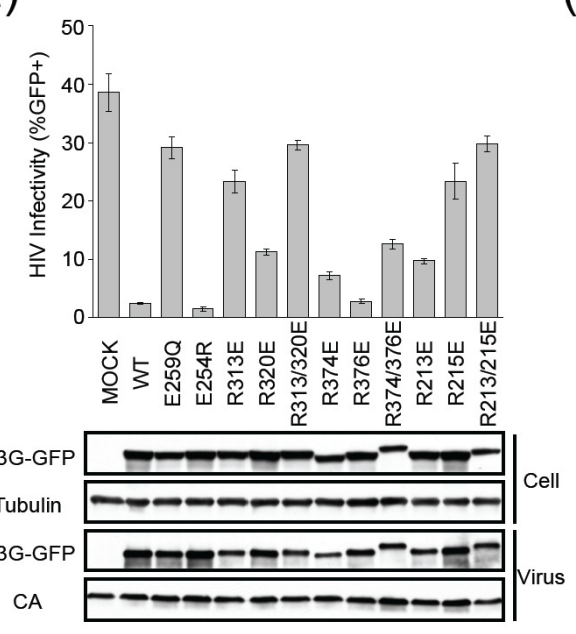

(b)

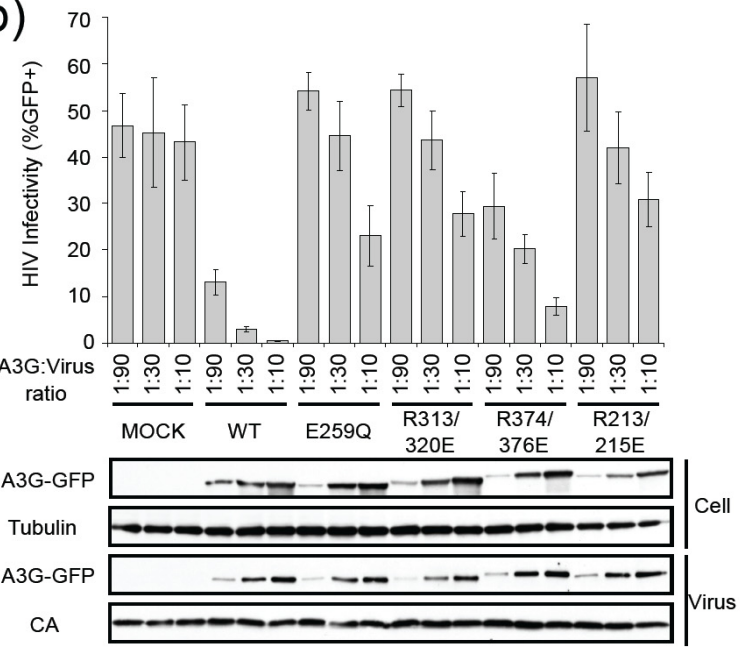

\subsection{DNA Binding Experiments}

The single-stranded DNA binding activity of A3G can be visualized by standard native gel EMSAs with labeled ssDNA [14,38-40]. We therefore used EMSAs to ask whether any of the aforementioned single mutants or R-to-E pairs altered the ssDNA binding activity of A3G. All of the proteins showed strong and almost indistinguishable ssDNA binding activity, even in dose response experiments (Figure 4a,b). These results could be rationalized, however, because the N-terminal (Z2) domain is thought to make the largest contribution to DNA binding. For instance, the apparent equilibrium dissociation constant $(\mathrm{Kd})$ for full-length $\mathrm{A} 3 \mathrm{G}$ to ssDNA is between 52 and $238 \mathrm{nM}[11,14,36]$, while that of the C-terminal domain alone is between 130 and $450 \mu \mathrm{M}[13,16]$.

Therefore, we next sought to combine $\mathrm{Z} 2$ amino acid substitutions reported previously to alter the RNA binding and/or localization activity of $\mathrm{A} 3 \mathrm{G}$ and the aforementioned R-to-E pairs $[18,20,21,29,30,41-45]$. We note however that, as yet, there is no A3G mutant that is completely defective for binding RNA or ssDNA. First, A3G E67Q, W94L, and W127A mutants were expressed as STP fusion proteins in HEK293T cells and purified as described above. The E67Q protein had 
lower yield and similar co-purifying background bands (Figure 5a). In contrast, both W94L and W127A proteins had atypical levels of additional bands (Figure 5a). This contamination complicated initial analyses but further work was able to reveal at least one important point (below). We note that none of these mutants was fully defective for ssDNA binding by EMSA or ssDNA deaminase activity in vitro (Figures $5 b, c)$.

Figure 4. APOBEC3G catalytic domain mutant electrophoretic mobility shift assays (EMSAs). (a) Native gel EMSAs for wildtype A3G and the indicated mutants. (b) Dose response EMSAs for wildtype $\mathrm{A} 3 \mathrm{G}$ and the indicated mutants $(0.2,0.4,0.8$ or $1.5 \mathrm{pmol}$ protein incubated with 4 pmol oligo).

(a)

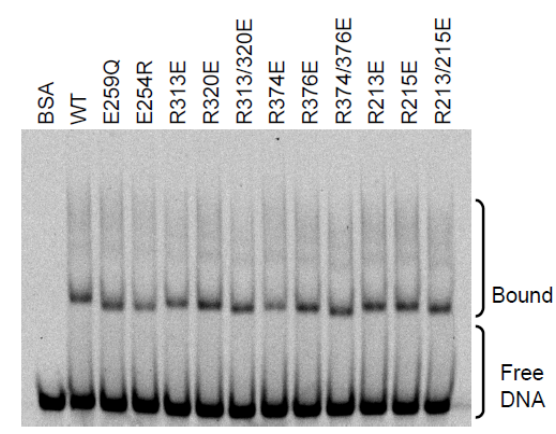

(b)

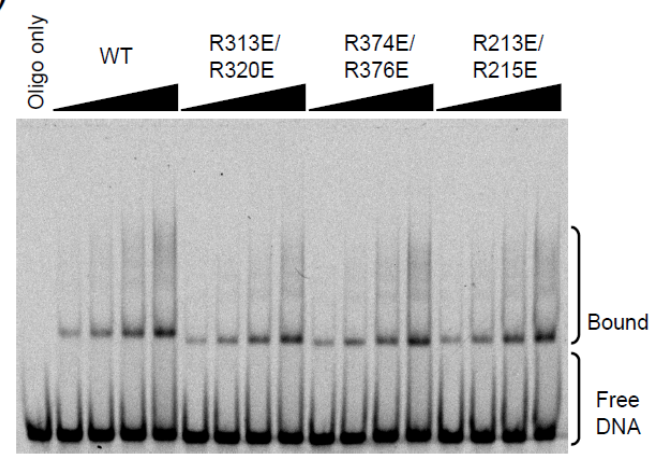

Figure 5. APOBEC3G N- and C-terminal domain mutant EMSAs. (a) Silver stained gel of protein samples used in ' $b$ ' and 'c'. BSA was used for quantification. (b) Native gel EMSAs for wildtype A3G and the indicated mutants. (c) Gel image of ssDNA deamination substrate and products produced by incubation with the indicated A3G proteins in vitro.

(a)

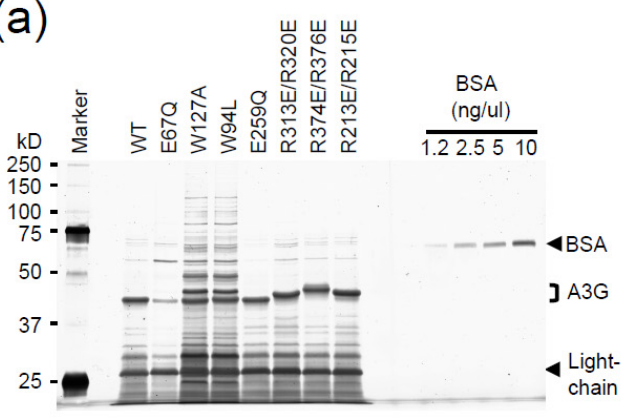

(b)

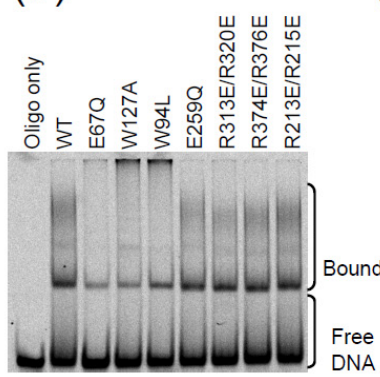

(c)

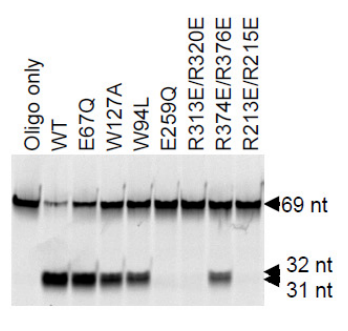

Next, the W94L and the W127A substitutions were combined with the R-to-E pairs R313E/R320E, $\mathrm{R} 213 \mathrm{E} / \mathrm{R} 215 \mathrm{E}$, and R374E/R376E to determine if the reduced DNA binding capacity of the $\mathrm{N}$-terminal domain substitutions could be exacerbated by C-terminal domain alterations (Figure 6a). As before, wildtype $\mathrm{A} 3 \mathrm{G}$ and proteins with each of the R-to-E pairs readily bound ssDNA and caused prominent gel shifts (Figure 6b). A3G with W127A or W94L substitutions had similar phenotypes, with each protein eliciting reduced levels of lower mass shifted bands and increased levels of higher mass shifted bands, suggesting an increased tendency to form protein/ssDNA aggregates. The ssDNA C-to-U deamination activities were as expected from above (Figure 6c). However, upon combination with the R313E/R320E substitutions (but not with R213E/R215E or R374E/R376E substitutions), the 
triple mutant proteins showed a significant reduction in lower but not upper mass shifted bands (Figure 6b). These data indicated for the first time by EMSA that the loop defined by R313 and R320 does indeed make a measurable contribution to the ssDNA binding activity of A3G.

Figure 6. APOBEC3G combination $\mathrm{N}$ - and C-terminal domain mutant EMSAs. (a) Silver stained gel of protein samples used in ' $b$ ' and ' $c$ '. BSA was used for quantification. An anti-strep immunoblot (below) was used to confirm similar levels of A3G-S protein in each lane. (b) Native gel EMSAs for wildtype A3G and the indicated mutants. Note the exacerbation of the EMSA phenotype of the W127A or W94L mutant in combination with R313E/R320E. (c) Gel image of ssDNA deamination substrate and products produced by incubation with the indicated $\mathrm{A} 3 \mathrm{G}$ proteins in vitro.

(a)

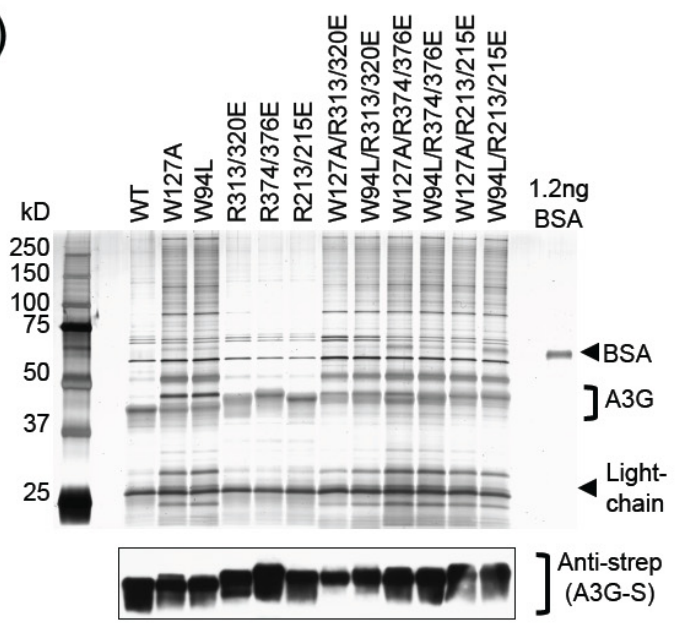

(b)

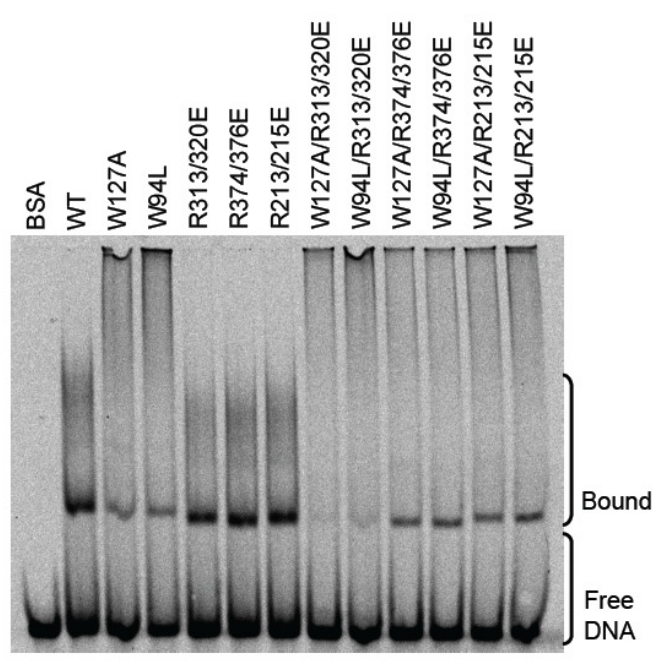

(c)

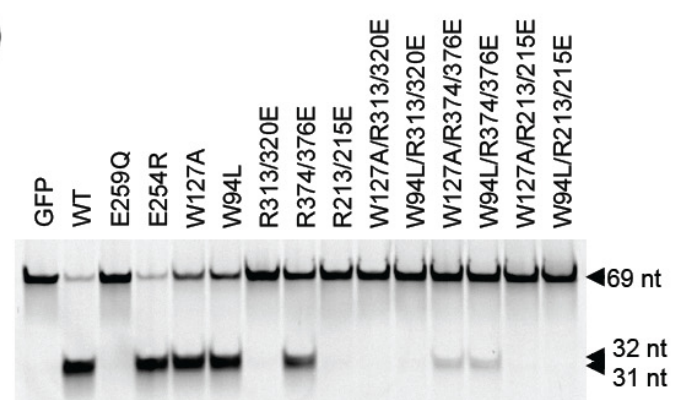




\subsection{NMR Chemical Shift Perturbations}

We next ${ }^{15} \mathrm{~N}$-labeled A3G191-384-2K3A [17,27] and performed a series chemical shift perturbation experiments to investigate potentially subtler catalytic domain-ssDNA contacts that may have eluded detection by EMSA. A number of residues showed significant chemical shifts (Table 1 and Supplementary Figure S1). Residues with shifts greater than $0.02 \mathrm{ppm}$ in ${ }^{1} \mathrm{H}$ or $0.2 \mathrm{ppm}$ in ${ }^{15} \mathrm{~N}$ are shaded blue and residues with lesser but continuous shifts are shown in green in Figure 7. Shifted residues indicative of contacts with ssDNA are positioned mostly on one surface, and together they comprise a near-continuous channel starting at loop 1, continuing through the active site region loops 2 and 3, and ending near the end of helix 2. These results are similar to two NMR titration data sets reported previously, but the chemical shift perturbations are much more localized here, owing to greater stability of the N-terminal region including helix 1 in this construct relative to shorter constructs used previously $[13,16,27]$. The channel defined by chemical shifts is most likely used for binding ssDNA by the C-terminal domain of A3G. It should be noted that some chemical shift changes were observed for the helix 6 region (residues 371-380). Finally, we note that both the N-terminal half and full-length $\mathrm{A} 3 \mathrm{G}$ can not be interrogated by this approach because they are insoluble at high concentrations.

Figure 7. NMR chemical shift perturbation map for APOBEC3G catalytic domain and single-stranded DNA. A space filled schematic of A3G191-384-2K3A with significant ssDNA chemical shift perturbations shown. Active site residues are colored magenta, strong chemical shifts blue, intermediate chemical shifts green, and all other residues gray. See Table 1 for raw data. (a) Active site surface and (b) a $180^{\circ}$ horizontal rotation from 'a'.

(a)

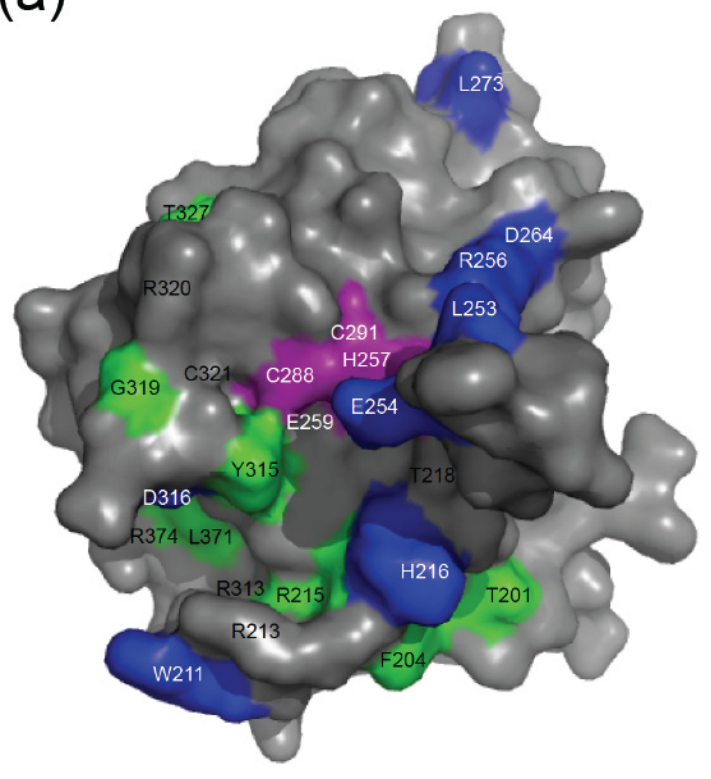

(b)

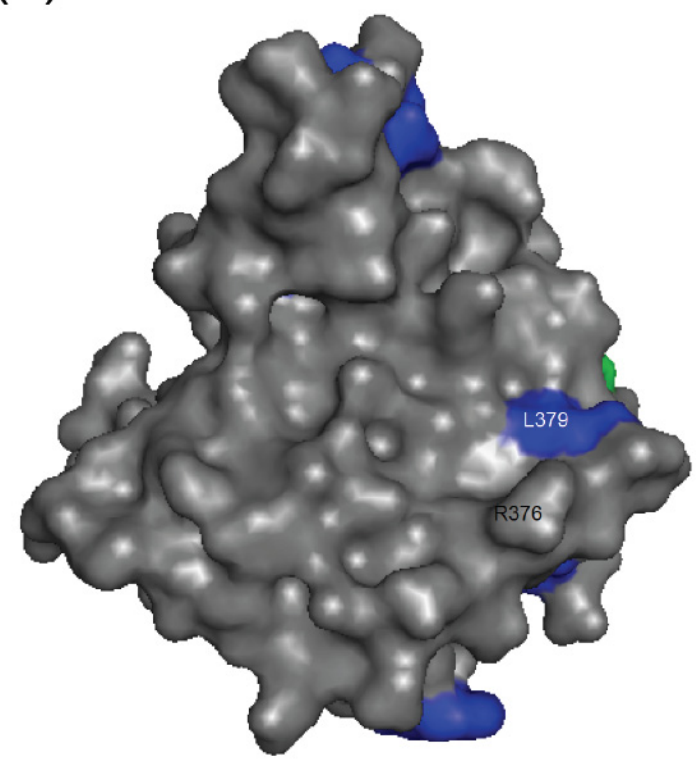


Table 1. Chemical shift data for A3G191-384-2K3A and $8 \times$ ssDNA.

\begin{tabular}{llll}
\hline Amino acid number & Shift in ${ }^{\mathbf{1}} \mathbf{H}$ (ppm) & Shift in ${ }^{\mathbf{1 5}} \mathbf{N}$ (ppm) & Combined Shift (ppm) \\
\hline 201 & 0.012 & 0.025 & 0.013 \\
204 & 0.014 & 0.051 & 0.017 \\
211 side chain & 0.038 & 0.137 & 0.047 \\
215 & 0.012 & 0.009 & 0.012 \\
216 & 0.023 & 0.137 & 0.035 \\
224 & 0.013 & 0.00 & 0.013 \\
253 & 0.028 & 0.256 & 0.059 \\
254 & 0.019 & 0.137 & 0.033 \\
256 & 0.028 & 0.155 & 0.042 \\
264 & 0.024 & 0.00 & 0.024 \\
273 & 0.012 & 0.222 & 0.046 \\
275 & 0.024 & 0.093 & 0.030 \\
276 & 0.016 & 0.144 & 0.033 \\
284 & 0.008 & 0.00 & 0.008 \\
313 & 0.008 & 0.004 & 0.010 \\
315 & 0.016 & 0.007 & 0.016 \\
316 & 0.051 & 0.128 & 0.057 \\
319 & 0.016 & 0.00 & 0.016 \\
327 & 0.011 & 0.00 & 0.011 \\
371 & 0.015 & 0.009 & 0.015 \\
374 & 0.007 & 0.00 & 0.007 \\
376 & 0.00 & 0.00 & 0.00 \\
378 & 0.012 & 0.00 & 0.012 \\
379 & 0.017 & 0.110 & 0.028 \\
\hline
\end{tabular}

\subsection{Discussion}

This is the first study to systematically mutagenize the purported DNA binding surface of the A3G catalytic domain and simultaneously assess the relative importance of key surface residues to deamination activity, HIV restriction activity, and ssDNA binding activity. Importantly, concordant with prior studies, deaminase activity can be completely abrogated with no measurable impact on ssDNA binding activity (e.g., E259Q, R215E, R313E; analogous data not shown for W285A). Such observations have been puzzling because, intuitively, the C-terminal domain $\underline{\text { must }}$ bind also ssDNA to correctly position the target cytosine in the active site for deamination. Therefore, it was satisfying to find that the ssDNA binding capacity of A3G could be diminished by R313E/R320E upon weakening the binding capacity of the N-terminal domain by substitutions at W94L or W127A.

The initial rationale for this study was to distinguish between Brim and Kink models for ssDNA binding and deamination by $\mathrm{A} 3 \mathrm{G}$. In order of weakest to strongest deaminase activity and HIV restriction phenotypes, $\mathrm{R} 313 \mathrm{E}=\mathrm{R} 215 \mathrm{E}=\mathrm{E} 259 \mathrm{Q}$ (severely compromised) $>\mathrm{R} 213 \mathrm{E}=\mathrm{R} 320 \mathrm{E}$ (intermediate) $>$ R374E (intermediate to weak) $>$ R376E $=$ E254R (weak to none; indistinguishable from wildtype $\mathrm{A} 3 \mathrm{G})$. These observations are most consistent with the Brim model. They also emphasize the importance of catalytic activity in HIV-1 restriction. However, many open questions remain such as how the holoenzyme binds polynucleotide substrates; how does polynucleotide binding 
influence oligomerization (and vice versa); are there any differences between ssDNA and RNA binding; and why are ssDNA but not RNA cytosines susceptible to deamination. Crystal structures of enzyme/substrate complexes and single molecule studies may shed light on these questions.

A3G is a potent DNA deaminase with strong RNA and ssDNA binding capabilities. Both of these activities are required for HIV restriction, with RNA binding required for encapsidation and ssDNA binding for efficient deamination. This study concerns the latter activity by suggesting a model in which initial high affinity contacts with ssDNA are made by N-terminal residues including W94 and W127 and then secondary, lower affinity contacts are made by C-terminal residues, particularly R313 and R215, to position ssDNA substrates for deamination (Figure 8). It is possible that RNA and ssDNA binding activities are governed by the same set of amino acid residues, but this then begs the question of how $\mathrm{A} 3 \mathrm{G}$ switches from binding RNA during encapsidation to binding ssDNA during reverse transcription. Competition experiments may shed light on this open question.

Figure 8. Two-step model for DNA binding and catalysis by A3G.

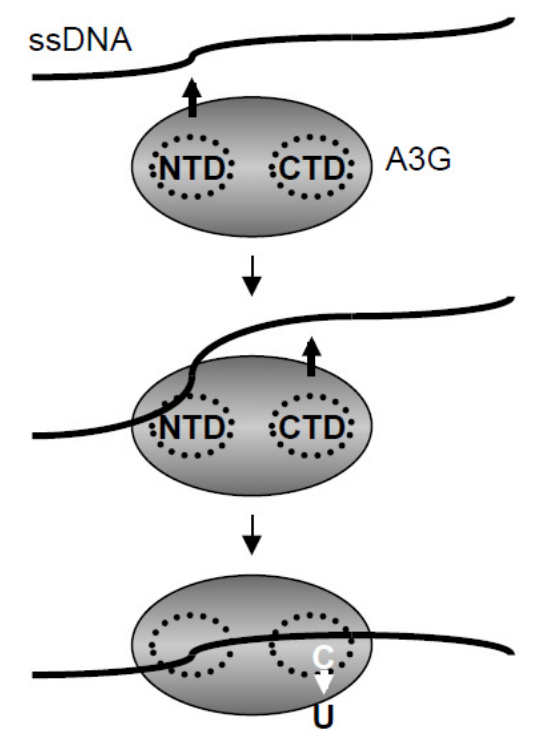

\section{Methods}

\subsection{Plasmid Construction}

pTrc99A-A3G was described previously [8]. R213E, R215E, E259Q, R313E, R320E, R374E, R376E, R213/215E, R313/320E, and R374/376E derivatives were generated by PCR-based site-directed mutagenesis. A3G-GFP was described previously [18,46]. R213E, R215E, E254R, E259Q, R313E, R320E, R374E, R376E, R213/215E, R313/320E, R374/376E, E67Q, W127A, W94L, W127A/R313/320E, W94L/R313/320E, W127A/R374/376E, W94L/R374/376E, W127A/R213/215E and W94L/R213/215E derivative mutants were generated by PCR-based site-directed mutagenesis. The pcDNA3-STP construct was generated by inserting sequences for the Strep epitope, the cleavage site of the Tobacco Etch Virus (TEV) protease, and the immunoglobulin binding domain of Protein A into pcDNA3.1 (Invitrogen) between the XhoI/XbaI sites. A3G-STP and derivative mutants were generated by inserting KpnI/SalI flanked A3G cDNA fragments from A3G-GFP and derivatives into $\mathrm{KpnI} / \mathrm{XhoI}$-digested pDNA3-STP. DNA sequencing was used to verify all constructs. 


\subsection{E. coli-Based DNA Deamination Assays}

General procedures for this assay were described [8]. For each condition, 8 single colonies were grown overnight at $37^{\circ} \mathrm{C}$ in LB medium containing $100 \mu \mathrm{g} / \mathrm{mL}$ ampicillin. Appropriate dilutions of cells were then spread on plates containing $100 \mu \mathrm{g} / \mathrm{mL}$ rifampicin, to select for rifampicin-resistant mutants and on plates containing $100 \mu \mathrm{g} / \mathrm{mL}$ ampicillin, to determine the number of viable cells. Mutation frequencies were calculated as the number of rifampicin-resistant mutants per $10^{7}$ viable cells. For immunoblots, cells were directly lysed by SDS sample buffer, resolved on 10\% SDS-polyacrylamide gel, transferred to a PVDF membrane (Millipore), and probed with an anti-A3G polyclonal antibody (NIH ARRRP10201).

\subsection{HIV-Restriction Assays and Immunoblots}

HIV-GFP reporter viruses were produced by transfection of HEK293T cells on 6-well plates with a four plasmid viral cocktail and A3G-GFP or its mutant derivatives or a control GFP construct using TransIT-LTI (Mirus) [47]. The HIV-GFP proviral plasmid CS-CG, the Gag-Pol expression plasmid, the Rev expression plasmid, and the VSV-G envelope expression plasmid constituted $0.9 \mu \mathrm{g}$ of the cocktail, and the vector control or the A3G expression plasmid constituted another $0.01 \mu \mathrm{g}$ (1:90), $0.03 \mu \mathrm{g}$ (1:30) or $0.09 \mu \mathrm{g}$ (1:10). The total amount of transfected DNA was adjusted to $1 \mu \mathrm{g}$ by adding pcDNA3.1 (Invitrogen). After 44hr-incubation, virus-containing supernatants were harvested through $0.22 \mu \mathrm{M}$ pore PVDF filter (Millipore). For HIV-restriction assays, $500 \mu \mathrm{L}$ of each supernatant was used to challenge fresh $5 \times 10^{4}$ HEK293T cells, and rates of GFP positive cells were scored by flow cytometry 2 days later. For immunoblots, $1 \mathrm{ml}$ of each supernatant was purified by centrifugation at $100,000 \mathrm{~g}$ for $1 \mathrm{~h}$ through a $20 \%$ sucrose containing PBS cushion. The resulting viral pellet was washed with PBS containing 20\% sucrose and then resuspended in SDS gel loading buffer, resolved on $7.5 \%$ (A3G-GFP and Tubulin) or 15\% (p24) SDS-PAGE gels, transferred to a PVDF membrane (Millipore), and probed with an anti-GFP antibody JL-8 (Invitrogen) to detect A3G-GFP or with an anti-p24 monoclonal antibody to detect viral Gag protein [48] (NIH ARRRP 6457). Both monoclonal antibodies were detected using a horseradish-peroxidase-conjugated goat anti-mouse IgG (Bio-Rad), followed by chemiluminescent imaging (Roche). After harvest of viral supernatants, A3G levels in virus-producing cells were monitored by extracting soluble proteins with RIPA buffer $\left(1 \mathrm{~h}, 4{ }^{\circ} \mathrm{C}\right.$, gentle rotation), removing particulates by centrifugation (10 $\mathrm{min}, 20,000 \mathrm{~g})$, and immunoblotting, as described above. An anti-tubulin monoclonal antibody (Covance) was used as cellular lysate loading control.

\subsection{Protein Expression and Purification}

A3G-STP, its mutant derivates, and control GFP-STP protein were expressed in HEK293T cells by transfection with $5 \mu \mathrm{g}$ of each construct using TransIT (Mirus). After $44 \mathrm{~h}$, cells were harvested by washing with PBS and then lysed with RIPA buffer supplemented with protease inhibitor cocktail (Roche), $40 \mathrm{mg} / \mathrm{mL}$ RNase A (Qiagen) and $1 \mathrm{U} / \mathrm{mL}$ DNase I. Sequential purification procedures were performed at $4{ }^{\circ} \mathrm{C}$ to minimize proteolysis and aggregation. After $1 \mathrm{~h}$ incubation, lysates were centrifuged at $20,000 \times \mathrm{g}$ for $10 \mathrm{~min}$ to remove insoluble fraction, then supernatants were mixed 
with $20 \mu \mathrm{L}$ bed volume of Ig sepharose (GE healthcare) and incubated for $3 \mathrm{~h}$. Beads were washed 4 times with RIPA buffer supplemented with protease inhibitor cocktail, and then washed 4 times with EMSA buffer (10 mM Tris-HCl, pH 7.5, $50 \mathrm{mM} \mathrm{NaCl}, 0.5 \%$ NP-40, 4\% glycerol, $1 \mathrm{mM} \mathrm{MgCl}_{2}$, $0.5 \mathrm{mM}$ EDTA and $1 \mathrm{mM}$ DTT). Cleavage reaction by AcTEV protease (Invitrogen) was performed in $300 \mu \mathrm{L}$ EMSA buffer for $16 \mathrm{~h}$. After short centrifugation, supernatants were transferred to new tubes and immediately tested for ssDNA binding and C-to-U deamination activities. Aliquots were snap frozen in liquid nitrogen and stored at $-80^{\circ} \mathrm{C}$.

\subsection{EMSAs}

Each reaction was performed by incubating $0.5-4$ pmol protein and 4 pmol Alexa ${ }^{488}$-labeled oligodeoxynucleotide (Alexa ${ }^{488}$-GAA-GAG-GAA-GGG-AAG-AAA-GAG-AAA-GGG-AGA-CCCAAA-GAG-GAA-AGG-TGA-GGA-GGT-TAA-TTT-GTG-TAA-ATA) in $20 \mu \mathrm{L}$ EMSA buffer for $20 \mathrm{~min}$ at room temperature. This 69 mer was reported previously [11]. Samples were made by adding $2.2 \mu \mathrm{L} 10 \times$ loading buffer $(250 \mathrm{mM}$ Tris- $\mathrm{HCl}, \mathrm{pH} 7.5,0.2 \%$ bromophenol blue and $40 \%$ glycerol) and resolved on $4 \%$ polyacrylamide-TBE gel at $4{ }^{\circ} \mathrm{C}$ and then fluorescence from the gel with wave length longer than $520 \mathrm{~nm}$ was detected by excitation at $450 \mathrm{~nm}$, using a Storm 840 imaging system (Molecular Dynamics).

\subsection{In Vitro DNA Deamination Assays}

16 pmol Alexa 488-labeled oligodeoxynucleotide (above) was incubated with $0.5-2$ pmol protein for $30 \mathrm{~min}$ in $10 \mu \mathrm{L}$ EMSA buffer, then with $5 \mathrm{U}$ uracil-DNA glycosylase (NEB) for 15 min and then mixed with $0.5 \mu \mathrm{L} 4 \mathrm{M} \mathrm{NaOH}$ and incubated for $10 \mathrm{~min}$ at $37^{\circ} \mathrm{C}$. After adding equal volume of formamide sample buffer, samples were resolved on 10\% polyacrylamide-TBE-7M-urea gel, and fluorescence was detected with a Storm 840 imaging system (Molecular Dynamics).

\subsection{NMR Chemical Shift Perturbation Experiments}

A3G-191-384-2K3A was purified as described [27] and concentrated to $300 \mu \mathrm{M}$ in phosphate buffer [1 mM dithiothreitol (DTT), $50 \mathrm{mM} \mathrm{Na} \mathrm{HPO}_{4} / \mathrm{NaH}_{2} \mathrm{PO}_{4}\left(\mathrm{pH}\right.$ 7.4) and $50 \mu \mathrm{M} \mathrm{ZnCl}_{2}$ ]. Non-labeled ssDNA 5'-GCT-TCT-TCT-ACC-TTC-TCT-TGA was hydrated in the same phosphate buffer and titrated into ${ }^{15} \mathrm{~N}$-labeled A3G-191-384-2K3A at DNA:protein molar ratios of 0:1, 4:1, 8:1, and 16:1. A heteronuclear single quantum coherence (HSQC) spectrum was recorded using a Bruker Megatron $700 \mathrm{MHz}$ Spectrometer at each molar ratio, which enabled quantification of amide proton chemical shift perturbations.

\section{Acknowledgements}

We thank J. Albin, M. Carpenter, J. Hultquist, A. Rathore, and N. Shaban for comments on this manuscript. This work was supported by NIH grants R01-AI064046 and P01-GM091743. The following reagents were obtained through the NIH AIDS Research and Reference Reagent Program, Division of AIDS, NIAID, NIH: anti-A3G antibody sera from Jaisri Lingappa and anti-p24 Gag monoclonal antibody from Michael Malim. 


\section{References}

1. Conticello, S.G. The AID/APOBEC family of nucleic acid mutators. Genome Biol. 2008, 9, 229.

2. LaRue, R.S.; Andresdottir, V.; Blanchard, Y.; Conticello, S.G.; Derse, D.; Emerman, M.; Greene, W.C.; Jonsson, S.R.; Landau, N.R.; Lochelt, M.; et al. Guidelines for naming nonprimate APOBEC3 genes and proteins. J. Virol. 2009, 83, 494-497.

3. Albin, J.S.; Harris, R.S. Interactions of host APOBEC3 restriction factors with HIV-1 in vivo: implications for therapeutics. Expert. Rev. Mol. Med. 2010, 12, e4.

4. Malim, M.H.; Emerman, M. HIV-1 accessory proteins-Ensuring viral survival in a hostile environment. Cell Host Microbe 2008, 3, 388-398.

5. Smith, J.L.; Bu, W.; Burdick, R.C.; Pathak, V.K. Multiple ways of targeting APOBEC3-virion infectivity factor interactions for anti-HIV-1 drug development. Trends. Pharmacol. Sci. 2009, 30, 638-646.

6. Hultquist, J.F.; Lengyel, J.A.; Refsland, E.W.; LaRue, R.S.; Lackey, L.; Brown, W.L.; Harris, R.S. Human and rhesus APOBEC3D, APOBEC3F, APOBEC3G, and APOBEC3H demonstrate a conserved capacity to restrict Vif-deficient HIV-1. J. Virol. 2011, 85, 11220-11234.

7. Refsland, E.W.; Hultquist, J.F.; Harris, R.S. Endogenous origins of HIV-1 G-to-A hypermutation and restriction in the nonpermissive T cell line CEM2n. PLoS Pathog. 2012, in press.

8. Harris, R.S.; Petersen-Mahrt, S.K.; Neuberger, M.S. RNA editing enzyme APOBEC1 and some of its homologs can act as DNA mutators. Mol. Cell 2002, 10, 1247-1253.

9. Jarmuz, A.; Chester, A.; Bayliss, J.; Gisbourne, J.; Dunham, I.; Scott, J.; Navaratnam, N. An anthropoid-specific locus of orphan $\mathrm{C}$ to U RNA-editing enzymes on chromosome 22. Genomics 2002, 79, 285-296.

10. Sheehy, A.M.; Gaddis, N.C.; Choi, J.D.; Malim, M.H. Isolation of a human gene that inhibits HIV-1 infection and is suppressed by the viral Vif protein. Nature 2002, 418, 646-650.

11. Chelico, L.; Pham, P.; Calabrese, P.; Goodman, M.F. APOBEC3G DNA deaminase acts processively 3' --> 5' on single-stranded DNA. Nat. Struct. Mol. Biol. 2006, 13, 392-399.

12. Holden, L.G.; Prochnow, C.; Chang, Y.P.; Bransteitter, R.; Chelico, L.; Sen, U.; Stevens, R.C.; Goodman, M.F.; Chen, X.S. Crystal structure of the anti-viral APOBEC3G catalytic domain and functional implications. Nature 2008, 456, 121-124.

13. Furukawa, A.; Nagata, T.; Matsugami, A.; Habu, Y.; Sugiyama, R.; Hayashi, F.; Kobayashi, N.; Yokoyama, S.; Takaku, H.; Katahira, M. Structure, interaction and real-time monitoring of the enzymatic reaction of wild-type APOBEC3G. EMBO J. 2009, 28, 440-451.

14. Iwatani, Y.; Takeuchi, H.; Strebel, K.; Levin, J.G. Biochemical activities of highly purified, catalytically active human APOBEC3G: Correlation with antiviral effect. J. Virol. 2006, 80, 5992-6002.

15. Harris, R.S.; Bishop, K.N.; Sheehy, A.M.; Craig, H.M.; Petersen-Mahrt, S.K.; Watt, I.N.; Neuberger, M.S.; Malim, M.H. DNA deamination mediates innate immunity to retroviral infection. Cell 2003, 113, 803-809.

16. Chen, K.M.; Harjes, E.; Gross, P.J.; Fahmy, A.; Lu, Y.; Shindo, K.; Harris, R.S.; Matsuo, H. Structure of the DNA deaminase domain of the HIV-1 restriction factor APOBEC3G. Nature 2008, 452, 116-119. 
17. Shandilya, S.M.D.; Nalam, M.N.L.; Nalivaika, E.A.; Gross, P.J.; Valesano, J.C.; Shindo, K.; Li, M.; Munson, M.; Royer, W.E.; Harjes, E.; et al. Crystal structure of the APOBEC3G catalytic domain reveals potential oligomerization interfaces. Structure 2010, 18, 28-38.

18. Stenglein, M.D.; Matsuo, H.; Harris, R.S. Two regions within the amino-terminal half of APOBEC3G cooperate to determine cytoplasmic localization. J. Virol. 2008, 82, 9591-9599.

19. Xu, H.; Svarovskaia, E.S.; Barr, R.; Zhang, Y.; Khan, M.A.; Strebel, K.; Pathak, V.K. A single amino acid substitution in human APOBEC3G antiretroviral enzyme confers resistance to HIV-1 virion infectivity factor-induced depletion. Proc. Natl. Acad. Sci. U. S. A. 2004, 101, 5652-5657.

20. Navarro, F.; Bollman, B.; Chen, H.; Konig, R.; Yu, Q.; Chiles, K.; Landau, N.R. Complementary function of the two catalytic domains of APOBEC3G. Virology 2005, 333, 374-386.

21. Bennett, R.P.; Presnyak, V.; Wedekind, J.E.; Smith, H.C. Nuclear Exclusion of the HIV-1 host defense factor APOBEC3G requires a novel cytoplasmic retention signal and is not dependent on RNA binding. J. Biol. Chem. 2008, 283, 7320-7327.

22. Haché, G.; Liddament, M.T.; Harris, R.S. The retroviral hypermutation specificity of APOBEC3F and APOBEC3G is governed by the C-terminal DNA cytosine deaminase domain. J. Biol. Chem. 2005, 280, 10920-10924.

23. Iwatani, Y.; Chan, D.S.; Liu, L.; Yoshii, H.; Shibata, J.; Yamamoto, N.; Levin, J.G.; Gronenborn, A.M.; Sugiura, W. HIV-1 Vif-mediated ubiquitination/degradation of APOBEC3G involves four critical lysine residues in its C-terminal domain. Proc. Natl. Acad. Sci. U. S. A. 2009, 106, 19539-19544.

24. Kohli, R.M.; Abrams, S.R.; Gajula, K.S.; Maul, R.W.; Gearhart, P.J.; Stivers, J.T. A portable hot spot recognition loop transfers sequence preferences from APOBEC family members to activation-induced cytidine deaminase. J. Biol. Chem. 2009, 284, 22898-22904.

25. Carpenter, M.A.; Rajagurubandara, E.; Wijesinghe, P.; Bhagwat, A.S. Determinants of sequence-specificity within human AID and APOBEC3G. DNA Repair (Amst) 2010, 9, 579-587.

26. Schumacher, A.J.; Haché, G.; MacDuff, D.A.; Brown, W.L.; Harris, R.S. The DNA deaminase activity of human APOBEC3G is required for Ty1, MusD, and human immunodeficiency virus type 1 restriction. J. Virol. 2008, 82, 2652-2660.

27. Harjes, E.; Gross, P.J.; Chen, K.M.; Lu, Y.; Shindo, K.; Nowarski, R.; Gross, J.D.; Kotler, M.; Harris, R.S.; Matsuo, H. An extended structure of the APOBEC3G catalytic domain suggests a unique holoenzyme model. J. Mol. Biol. 2009, 389, 819-832.

28. Li, M.; Shandilya, S.M.; Carpenter, M.A.; Rathore, A.; Brown, W.L.; Perkins, A.L.; Harki, D.A.; Solberg, J.; Hook, D.J.; Pandey, K.K.; et al. First-in-class small molecule inhibitors of the singlestrand DNA cytosine deaminase APOBEC3G. ACS Chem. Biol. 2012, 7, 506-517.

29. Newman, E.N.; Holmes, R.K.; Craig, H.M.; Klein, K.C.; Lingappa, J.R.; Malim, M.H.; Sheehy, A.M. Antiviral function of APOBEC $3 \mathrm{G}$ can be dissociated from cytidine deaminase activity. Curr. Biol. 2005, 15, 166-170.

30. Mangeat, B.; Turelli, P.; Caron, G.; Friedli, M.; Perrin, L.; Trono, D. Broad antiretroviral defence by human APOBEC3G through lethal editing of nascent reverse transcripts. Nature 2003, 424, 99-103. 
31. Shindo, K.; Takaori-Kondo, A.; Kobayashi, M.; Abudu, A.; Fukunaga, K.; Uchiyama, T. The enzymatic activity of CEM15/APOBEC3G is essential for the regulation of the infectivity of HIV-1 virion but not a sole determinant of its antiviral activity. J. Biol. Chem. 2003, 278, 44412-44416.

32. Haché, G.; Shindo, K.; Albin, J.S.; Harris, R.S. Evolution of HIV-1 isolates that use a novel Vifindependent mechanism to resist restriction by human APOBEC3G. Curr. Biol. 2008, 18, 819-824.

33. Browne, E.P.; Allers, C.; Landau, N.R. Restriction of HIV-1 by APOBEC3G is cytidine deaminase-dependent. Virology 2009, 387, 313-321.

34. Miyagi, E.; Opi, S.; Takeuchi, H.; Khan, M.; Goila-Gaur, R.; Kao, S.; Strebel, K. Enzymatically active APOBEC3G is required for efficient inhibition of human immunodeficiency virus type 1. J. Virol. 2007, 81, 13346-13353.

35. Holmes, R.K.; Koning, F.A.; Bishop, K.N.; Malim, M.H. APOBEC3F can inhibit the accumulation of HIV-1 reverse transcription products in the absence of hypermutation. Comparisons with APOBEC3G. J. Biol. Chem. 2007, 282, 2587-2595.

36. Iwatani, Y.; Chan, D.S.; Wang, F.; Maynard, K.S.; Sugiura, W.; Gronenborn, A.M.; Rouzina, I.; Williams, M.C.; Musier-Forsyth, K.; Levin, J.G. Deaminase-independent inhibition of HIV-1 reverse transcription by APOBEC3G. Nucleic Acids Res. 2007, 35, 7096-7108.

37. Bishop, K.N.; Verma, M.; Kim, E.Y.; Wolinsky, S.M.; Malim, M.H. APOBEC3G inhibits elongation of HIV-1 reverse transcripts. PLoS Pathog. 2008, 4, e1000231.

38. Yu, Q.; Konig, R.; Pillai, S.; Chiles, K.; Kearney, M.; Palmer, S.; Richman, D.; Coffin, J.M.; Landau, N.R. Single-strand specificity of APOBEC3G accounts for minus-strand deamination of the HIV genome. Nat. Struct. Mol. Biol. 2004, 11, 435-442.

39. McDougall, W.M.; Okany, C.; Smith, H.C. Deaminase activity on single-stranded DNA (ssDNA) occurs in vitro when APOBEC3G cytidine deaminase forms homotetramers and higher-order complexes. J. Biol. Chem. 2011, 286, 30655-30661.

40. Shlyakhtenko, L.S.; Lushnikov, A.Y.; Li, M.; Lackey, L.; Harris, R.S.; Lyubchenko, Y.L. Atomic force microscopy studies provide direct evidence for dimerization of the HIV restriction factor APOBEC3G. J. Biol. Chem. 2011, 286, 3387-3395.

41. Zhang, K.L.; Mangeat, B.; Ortiz, M.; Zoete, V.; Trono, D.; Telenti, A.; Michielin, O. Model structure of human APOBEC3G. PLoS One 2007, 2, e378.

42. Huthoff, H.; Autore, F.; Gallois-Montbrun, S.; Fraternali, F.; Malim, M.H. RNA-dependent oligomerization of APOBEC3G is required for restriction of HIV-1. PLoS Pathog. 2009, 5, e1000330.

43. Huthoff, H.; Malim, M.H. Cytidine deamination and resistance to retroviral infection: Towards a structural understanding of the APOBEC proteins. Virology 2005, 334, 147-153.

44. Friew, Y.N.; Boyko, V.; Hu, W.S.; Pathak, V.K. Intracellular interactions between APOBEC3G, RNA, and HIV-1 Gag: APOBEC3G multimerization is dependent on its association with RNA. Retrovirology 2009, 6, 56.

45. Opi, S.; Takeuchi, H.; Kao, S.; Khan, M.A.; Miyagi, E.; Goila-Gaur, R.; Iwatani, Y.; Levin, J.G.; Strebel, K. Monomeric APOBEC3G is catalytically active and has antiviral activity. J. Virol. 2006, 80, 4673-4682. 
46. Stenglein, M.D.; Harris, R.S. APOBEC3B and APOBEC3F inhibit L1 retrotransposition by a DNA deamination-independent mechanism. J. Biol. Chem. 2006, 281, 16837-16841.

47. Liddament, M.T.; Brown, W.L.; Schumacher, A.J.; Harris, R.S. APOBEC3F properties and hypermutation preferences indicate activity against HIV-1 in vivo. Curr. Biol. 2004, 14, $1385-1391$.

48. Simon, J.H.; Fouchier, R.A.; Southerling, T.E.; Guerra, C.B.; Grant, C.K.; Malim, M.H. The Vif and Gag proteins of human immunodeficiency virus type 1 colocalize in infected human $\mathrm{T}$ cells. J. Virol. 1997, 71, 5259-5267.

\section{Supplementary Materials}

Figure S1. Raw NMR spectroscopy chemical shift perturbation data. NMR chemical shift perturbation spectra for residues listed in table1. A3G-191-384-2K3A was incubated with a ssDNA (5'-GCT TCT TCT ACC TTC TCT TGA-3') at protein to ssDNA molar ratios of 1 to $0,1: 4,1: 8$ and 1:16 colored black, blue, green and red, respectively.
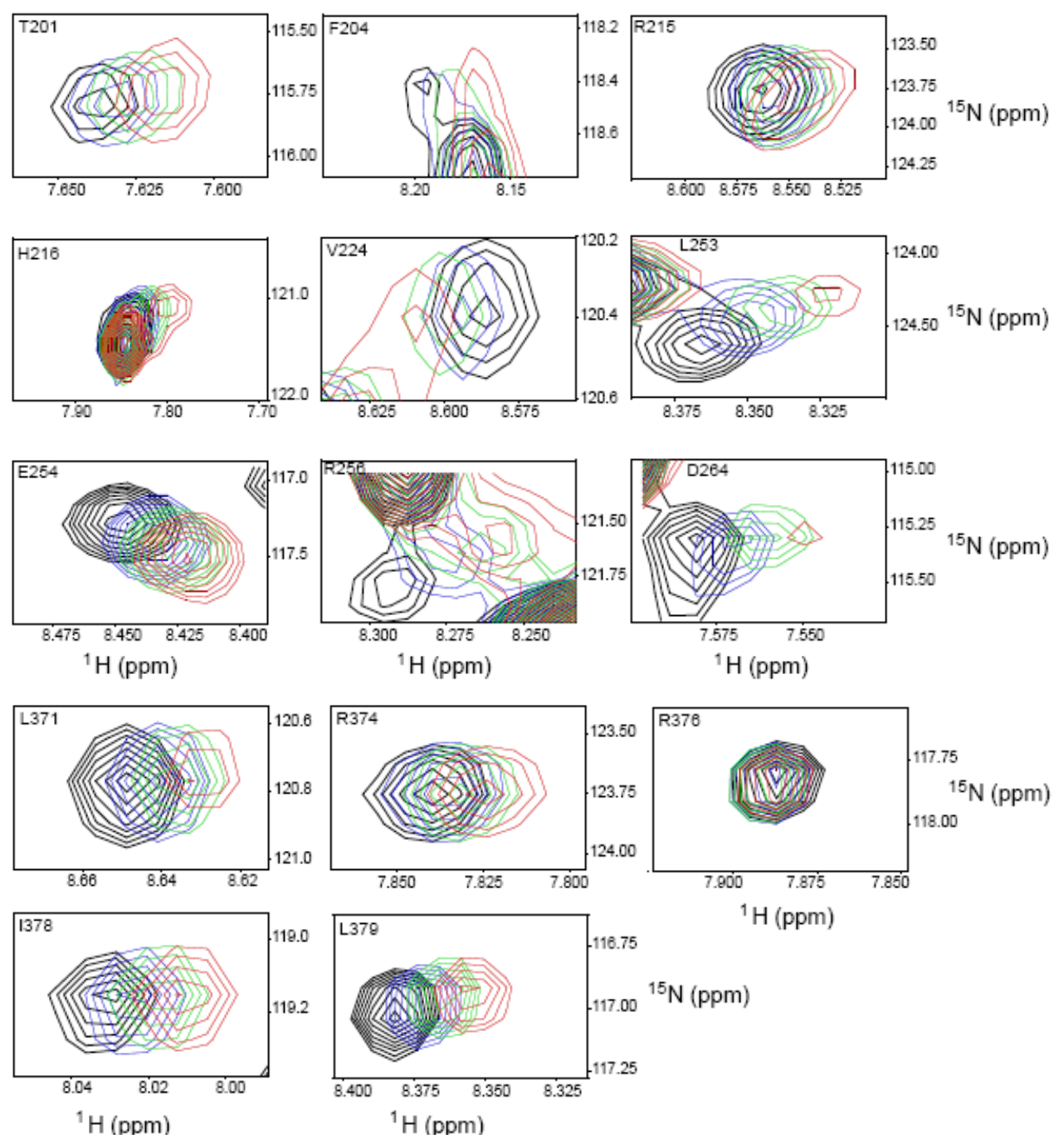

(C) 2012 by the authors; licensee MDPI, Basel, Switzerland. This article is an open access article distributed under the terms and conditions of the Creative Commons Attribution license (http://creativecommons.org/licenses/by/3.0/). 